\title{
Two major survival surgeries, one protocol: how should the IACUC report violations and change practices?
}

\author{
Jerald Silverman, DVM
}

To help make writing protocols easier for its faculty, the IACUC at Great Eastern University allowed for many separate experiments to be included on the same protocol, as long as the general theme of the research was the same. For example, a researcher may have amended his or her protocol many times over its threeyear lifespan to include multiple separate Parkinson's disease treatment studies. The rationale used by the IACUC was that the studies were all Parkinson's treatmentrelated, even if the basic questions being asked differed. As expected, some protocols became quite large and difficult to review, but the investigators were pleased that they could just add another study or technique to an existing protocol without having to write an entirely new one.

Dr. Wanda White used cynomolgus monkeys for her Parkinson's disease research. Her basic methodology included the chemical induction of Parkinson's-like disease and a surgical procedure to make a craniotomy for subsequent brain activity recording. After the study was completed, White's lab maintained the animals until clinical signs lead to significant deterioration of the animals' well-being, and they were then euthanized. Moving into a related research area, White began studying the protein alphasynuclein in intestinal nerve cells and its relationship to intestinal problems associated with Parkinson's disease. Her initial experiments required a major survival surgical procedure to isolate and remove segments of certain intestinal nerves. The animals were euthanized one week postoperatively. She received IACUC approval for the procedure as an amendment to the same protocol that also contained the craniotomy procedure. For the intestinal surgery, she used one of the animals that previously had undergone a craniotomy. This was quickly recognized by the vivarium staff and brought to the IACUC as a violation of federal regulations that prohibit the use of the same animal for two unrelated survival surgical procedures. The committee told White that on her protocol application she had checked the box that indicated she would not use the same animal for two major survival surgical procedures without IACUC approval, yet she did just that. White apologized and said that it was a postdoctoral associate who had written the protocol and although she reviewed it, she simply missed that checked box and she used the same animal to save time and money. Dr. Larry Covello, the IACUC chairman, noted that although the same animal may not be used in two separate protocols for major survival operative procedures, in this case the animals were actually part of the same protocol, so perhaps there was no violation of that particular federal regulation even though there was noncompliance with her approved protocol.

How do you think this problem should be reported to the USDA and NIH, and what could be done to prevent similar problems in the future?

\section{RESPONSE}

\section{Oops!... I did it again}

\section{Jori K Leszczynski, Derek Fong, Christopher Manuel \& Michelle Wallace}

While there is not a regulatory limit to the number of procedures in an IACUC proto$\mathrm{col}$, the Great Eastern IACUC should ensure that they understand how procedures are interrelated and what procedures are performed on an individual animal. White is not that innocent in regards to compliance with her protocol. Even if it was a mistake in oversight, she clearly did not indicate that animals would undergo multiple major survival surgeries. While the IACUC could have clarified this issue during its review, it is ultimately White's responsibility to ensure that the protocol accurately describes what will be performed on each animal. In addition, her stated justification for performing multiple major survival surgical procedures is inadequate according to the Guide ${ }^{1}$ and the Animal Welfare Regulations and Policies $^{2,3}$, as all of these require a scientific justification or clinical necessity. They also specify that the surgeries must be essential components of a single research project or protocol. Although the surgeries are on the same protocol, they are not interdependent. At this point, there is no justification in the protocol and when questioned as to why this was necessary, White only stated she was trying to save time and money. If she would like to do this in the future, she will need to provide a scientific justification to the IACUC, which includes how the two procedures are interrelated. The only other option for White would be for the Institutional Official to appeal to the USDA Animal Care Regional Director who will forward it to the Animal Care Deputy Administrator for consideration of a special circumstance. This appeal would need to include several pieces of information: a scientific justification that does not include cost, the procedures proposed, and assurance that the IACUC has approved this exemption ${ }^{2,3}$.

If White is Public Health Service (PHS) funded and Great Eastern has an assurance on file with OLAW, the IACUC must report this non-compliance to OLAW. According to OLAW's Guidance on Prompt Reporting ${ }^{4}$, it would meet the criteria of conduct of 